\title{
The Effect of Thyroid Dysfunction on the Cardiovascular Risk of Type 2 Diabetes Mellitus Patients in Ghana
}

\author{
Osei Sarfo-Kantanka $\mathbb{D}^{1},{ }^{1}$ Fred Stephen Sarfo, ${ }^{1,2}$ Eunice Oparebea Ansah, ${ }^{1}$ and Ishmael Kyei ${ }^{1}$ \\ ${ }^{1}$ Komfo Anokye Teaching Hospital, Kumasi, Ghana \\ ${ }^{2}$ Kwame Nkrumah University of Science and Technology, Kumasi, Ghana
}

Correspondence should be addressed to Osei Sarfo-Kantanka; osarfokantanka21@gmail.com

Received 5 July 2017; Revised 14 October 2017; Accepted 30 October 2017; Published 1 February 2018

Academic Editor: Eusebio Chiefari

Copyright (C) 2018 Osei Sarfo-Kantanka et al. This is an open access article distributed under the Creative Commons Attribution License, which permits unrestricted use, distribution, and reproduction in any medium, provided the original work is properly cited.

\begin{abstract}
Background. Thyroid dysfunction is known to exaggerate the coronary heart disease (CHD) risk associated with type 2 diabetes mellitus (T2DM) among whites. The effect is yet to be studied among African populations. Methods. This is a cross-sectional study involving 780 T2DM patients enrolled in a diabetes clinic in Kumasi, Ghana. CHD risk was estimated using the Framingham and UKPDS risk scores. Risks were categorised as low (<10\%), intermediate (10-19\%), and high $(\geq 20 \%)$. Associations between metabolic risk factors, thyroid dysfunction, and CHD risk were measured using Spearman's partial correlation analysis while controlling for age and gender. Differences were considered statistically significant at $p<0.05$. Results. $780 \mathrm{~T} 2 \mathrm{DM}$ patients $(57.7 \%$ females $)$, mean $\pm \mathrm{SD}$ age of $57.4 \pm 9.4$ was analysed. The median (IQR) 10-year CHD score estimated using the Framingham and UKPDS risk engines for males and females was 12 (8-20), 9.4 (5.7-13.4), $p<0.0001$ and 3 (1-6), 5.8 (3.4-9.6), $p<0.0001$, respectively. Positive correlation was found between CHD risk and HbA1c, total cholesterol, low-density lipoprotein cholesterol, systolic blood pressure, and thyroid stimulating hormone. Conclusion. The presence of thyroid dysfunction significantly increased the CHD risk associated with T2DM patients in Ghana.
\end{abstract}

\section{Introduction}

There has been a remarkable surge in the worldwide prevalence of type 2 diabetes mellitus (T2DM) over the past two decades. Per estimates by the International Diabetes Federation (IDF), at least 415 million people worldwide had diabetes mellitus in 2015 [1]. Countries in sub-Saharan Africa (SSA) will suffer significantly, with a projection of over 30 million cases expected to be recorded over the next three decades [2]. Ghana, like in most SSA countries, has been affected by profound escalations in the rates of all traditional vascular risk factors including diabetes, hypertension, dyslipidemia, and obesity [3]. Currently, an estimated $6 \%$ of Ghanaian urban population has been affected by diabetes [4].

Patients with diabetes compared to nondiabetic individuals bear up to six-fold higher risk of future coronary heart disease (CHD), equivalent to nondiabetic patients with preexisting cardiovascular disease [5-8]. Part of this excess risk found in diabetes patients is attributed to a higher prevalence of other cardiovascular disease (CVD) risk factors such as obesity, dyslipidemia, and hypertension.

Thyroid conditions represent the second commonest endocrine disorder seen in adults [9]. The coexistence of both T2DM and thyroid diseases can further increase the CHD risk associated with T2DM [10]. Like T2DM, thyroid disorders can have deleterious effects on the cardiovascular system; thyrotoxicosis leads to a hyperdynamic state with increased heart rate, left ventricular contractility, and systolic hypertension and may also be complicated by atrial fibrillation [11, 12]. Hypothyroidism, on the other hand, can enhance CHD risk through multiple interactions with indices like dyslipidemia and hypertension [13-16]. Identifying the CHD risk in T2DM patients with thyroid dysfunction will be beneficial in the end since evidence points to patients benefiting from aggressive strategies in CVD risk reduction [17]. 
Despite a well-known association of thyroid dysfunction with T2DM, no known studies have been conducted in subSaharan Africa to investigate the effect of such relationship on CHD risk of T2DM patients. Our objective in this study was to identify the effect of thyroid dysfunction on the CHD risk of Ghanaian T2DM patients attending an outpatient diabetes clinic using the well-validated Framingham risk [18] and the United Kingdom Prospective Diabetes Study Scores (UKPDS) [19].

\section{Methods}

2.1. Study Design and Setting. This study is a hospital-based cross-sectional study conducted at the Diabetes Clinic of the Komfo Anokye Teaching Hospital, a tertiary referral medical center in Kumasi, Ghana. Kumasi is the second largest city in Ghana, with an estimated population of 4 million inhabitants. The Diabetes Clinic was established in 1992 and runs throughout the working week receiving referrals for adults $>16$ years with diabetes disorders from 6 out of the 10 administrative regions of Ghana with an estimated population of 10 million.

2.2. Study Participants. Consecutive T2DM patients attending the Diabetes Clinic at KATH were approached for enrollment into the study after obtaining informed consent. Patients were diagnosed with T2DM when they fulfilled the World Health Organization (WHO) diagnostic criteria for diabetes: an elevated fasting plasma glucose level $(\geq 7 \mathrm{mmol} / \mathrm{L}$ ) on two occasions, or oral glucose tolerance test $\geq 11.1 \mathrm{mmol} / \mathrm{L}$ [20], and age 30 years or older at the time of diagnosis, had not undergone insulin therapy for a year after diagnosis, and had no history of diabetic ketoacidosis. Using a structured validated questionnaire and a review of medical records, we obtained the sociodemographic and clinical information of all participants. Because of their confounding effects on thyroid function, we excluded pregnant women, patients on amiodarone, lithium, and long-term corticosteroids, and those with an acute illness and history of hospitalisation less than six months from the day of recruitment.

2.3. Consent and Ethical Approval. We obtained ethical approval from the Committee on Human Research Publication and Ethics (CHRPE) of the School of Medical Sciences, Kwame Nkrumah University of Science and Technology, and the Komfo Anokye Teaching Hospital (KATH), Kumasi, Ghana. All participants gave an informed consent with those unable to understand or sign the consent forms excluded.

2.4. Study Measurements. Patients were interviewed using a well-validated questionnaire to obtain information such as age, gender, smoking, and alcohol consumption habits. Current smoking status and alcohol intake status were ascertained from either the patient or a responsible relative. Smokers were identified by self-report as those who had smoked at least 10 sticks of cigarettes per day for six months or more or those who smoked daily for one year or more regardless of the number of cigarettes smoked per day [21].
A high alcohol intake was defined as $\geq 14 \mathrm{U}$ per week for women and $\geq 21 \mathrm{U}$ per week for men [22].

2.5. Physical Measurements. The weight of study subjects was measured in kilograms using a scale, and the height in centimeters using a Stadiometer with the patient standing at the anatomical position. The weight and height measurements were used to calculate the BMI. Subjects with $\mathrm{BMI} \geq 30 \mathrm{~kg} / \mathrm{m}^{2}$ were classified as obese [23].

Duplicate waist circumference (WC) measurements were taken, and the average was recorded using a plastic stretch-resistant anthropometric tape calibrated in centimeters and inches (made in Shanghai, China). The measurements were taken from the approximate midpoint between the lower margin of the last palpable rib and the superior border of the iliac crest at the level of the umbilicus in the midaxillary line with the participant erect and abdomen relaxed, arms by the side and heels together, and breathing normally. WC measurements $>80 \mathrm{~cm}$ and $94 \mathrm{~cm}$ were recorded as central obesity for females and males, respectively [23].

Blood pressure was measured thrice on the upper left arm using a validated automatic sphygmomanometer after at least 5 minutes of rest and the second and third readings averaged for analysis. Hypertension was diagnosed if the patient was on antihypertensive medications or if the patient had a systolic and diastolic blood pressure of 140/90 mmHg [24].

2.6. Laboratory Measurements of Biochemical Variables. Approximately ten milliliters $(10 \mathrm{~mL})$ of fasting venous samples was collected from each participant into Vacutainer tubes (Becton Dickinson, Rutherford, NJ) and Sequestrene bottles. Samples were manually processed and cryopreserved before transporting to the laboratory for analysis. Fasting plasma glucose (FPG), free thyroxine (FT4), free triiodothyronine (FT3), thyroid stimulating hormone (TSH), total cholesterol (TC), low-density lipoprotein cholesterol (LDL-C), high-density lipoprotein cholesterol (HDL-C), triglycerides (TG), urea, and creatinine levels were analysed on the serum obtained from each participant using a Cobras e 411 autoanalyzer $^{\circledR}$ per manufacturer's instructions and previously described by Sarfo-Kantanka et al. [25].

Dyslipidemia was defined as a high TC, $>5 \mathrm{mmol} / \mathrm{L}$ or LDL-cholesterol $>4 \mathrm{mmol} / \mathrm{L}$, triglycerides $>1.7 \mathrm{mmol} / \mathrm{L}$, or HDL-cholesterol $<1.30 \mathrm{mmol} / \mathrm{L}$ for women and $<1.04 \mathrm{mmol} / \mathrm{L}$ for men or previous use of statin for dyslipidemia [26].

The reference ranges, intra-assay, and interassay coefficients of variation for thyroid hormones were as follows: TSH: $0.25-5.0 \mathrm{IU} / \mathrm{mL},<2.1 \%$ and $<2.4 \%$; FT3: 3.7-10.4 pmol/L, $5.8 \%$ and $6.9 \%$ for FT3; and FT4: 7.5-21.1 pmol/L, $2.8 \%$ and $2.4 \%$.

Thyroid function was classified as euthyroidism when free thyroxine (FT4), free triiodothyronine (FT3), and thyroid stimulating hormone (TSH) were within the normal range; clinical hypothyroidism when TSH level was greater than the upper limit of the reference range and FT4 or FT3 was lower than the lower limit of their reference ranges; subclinical hypothyroidism when TSH is greater 
than the upper limit of the reference range and FT4 and FT3 are within the normal range; clinical hyperthyroidism when TSH level was lower than the lower limit of the reference range and FT4 or FT3 was greater than the upper limit of their reference ranges; and subclinical hyperthyroidism when TSH level was lower than the lower limit of the reference range and FT3 and FT4 are within the normal range.

Glycated hemoglobin (HbAlc) measurements were performed using standardized high-performance liquid chromatography assay using Bio-Rad Variant. We followed the methodology described by Darko et al. [27].

2.7. Cardiovascular Risk Estimation. The Framingham risk engine and UKPDS questionnaire were administered to all eligible subjects and used to calculate the 10-year CHD risk of each participant. The Framingham risk questionnaire is a gender- and LDL-C-specific questionnaire and incorporates risk factors including age, sex, TC, HDL-C, SBP, DBP, smoking status, and presence of diabetes. UKPDS is an offline diabetes-specific risk engine used to estimate the 10-year CHD risk of an individual. The model is diabetes-specific and incorporates $\mathrm{HbAlc}$, SBP, and lipid levels as risk factors, in addition to age, sex, ethnic group, smoking status, and time since diagnosis of diabetes. For the UKPDS risk estimation, participants were treated as African Caribbean. Estimated CHD risks were then categorised as low $(<10 \%)$, intermediate $(10-20 \%)$, and high $(>20 \%)$.

2.8. Statistics. Data was entered into Microsoft Excel, and statistical analysis was performed using GraphPad Prism 5 software. Data normality was tested using D'Agostino and Pearson omnibus normality test. The statistical difference between means and medians was estimated by the Student $t$-tests and Mann-Whitney $U$ test. Associations were measured by coefficients of Spearman's partial correlation whiles controlling for age and gender. Differences were considered statistically significant at $p<0.05$.

\section{Results}

3.1. Demographic and Clinical Characteristics. 958 participants were approached, but 178 declined participation in the study. As shown in Table 1, 780 T2DM subjects, mean age $57.4 \pm 9.4$, were included in the analyses. Of these, 450 $(57.7 \%)$ subjects were female. The median (IQR) age at diagnosis of participants was 50 (41-54), and the mean duration of T2DM was $9.8 \pm 5.6$ years. The predominant vascular risk factors identified included hypertension (77.9\%), central obesity (74.6\%), dyslipidemia (59.2\%), and abnormal glycemic control, HbAlc>7\% (66.2\%). Females had a significantly higher prevalence of central obesity, high BMI, and DBP. The median (IQR) 10-year CHD score estimated using the Framingham and UKPDS risk engines for males and females was $12(8-20), 9.4$ (5.7-13.4), $p<0.0001$ and 3 (1-6), 5.8 (3.4-9.6), $p<0.0001$, respectively. The median (IQR) CHD risk score estimated using the UKPDS engine was 4 (0.4-7): 9.4 (5.7-13.4) for males and 5.8 (3.4-9.6), $p<0.0001$, for females.

3.2. CHD Score Based on Thyroid Status. Table 2 shows the median (IQR) Framingham risk score of T2DM patients with thyroid dysfunction significantly higher than for euthyroid T2DM patients: $11(6-22)$ versus $4(1-10), p<0.0001 .8 \%$ of euthyroid T2DM subjects were identified as having high CHD risk using the Framingham risk score, 24\% had intermediate risk, and $68 \%$ had low risk. Among those with thyroid dysfunction, $43 \%$ were identified as high risk, $28 \%$ as medium risk, and $25 \%$ as low risk. Of those identified with subclinical hyperthyroidism, 32\% had high risk of $\mathrm{CHD}, 23 \%$ had an intermediate risk, and $44 \%$ had low risk. Of those with hyperthyroidism, 36\% had high risk, $42 \%$ intermediate risk, and $22 \%$ low risk of CHD (Figure 1). In patients with subclinical hypothyroidism, $48 \%$ were classified as high risk, $29 \%$ as intermediate risk, and $23 \%$ as low risk; with hypothyroidism, $55 \%$ had high risk, $31 \%$ intermediate risk, and $14 \%$ low risk. The difference between the groups was highly significant $(p<0.0001)$. Using the UKPDS score, $4 \%$ of euthyroid T2DM subjects were identified as high risk, $44 \%$ as intermediate risk, and $52 \%$ as low risk. Among those with thyroid dysfunction, $26 \%$ were classified as high risk of $\mathrm{CHD}, 58 \%$ as intermediate risk, and $16 \%$ as low risk.

3.3. Correlates of Increased Cardiovascular Risk. As shown in Table 2, the demographic characteristics, vascular risk factor profile, and clinical features of T2DM subjects classified as having high $\mathrm{CHD}$ risk using the Framingham risk score were older, had female gender predisposition, older age at diagnosis, obese (general and central), had poorer glycemic control and lipid profiles, higher blood pressures, and higher rates of alcohol abuse compared to those with intermediate or low CHD risks.

3.3.1. Association between Biochemical and Physical Characteristics and Coronary Heart Disease Risk in T2DM Patients. A strong positive correlation was found between high Framingham CHD risk and HbAlc $(r=0.51, p<0.04)$, TC $(r=0.49, p<0.0001)$, LDL-C $(r=0.37, p<0.0001)$, and TSH $(r=0.27, p=0.01)$. Under the UKPDS scoring, a strong association was found between CHD risk and HbAlc $(r=0.42, p<0.0001)$ and TSH $(r=0.32, p<0.02)$ (Table 3$)$.

\section{Discussion}

We have found in this study that T2DM subjects in Ghana possess high prevalence levels of modifiable vascular risk factors: $74.5 \%$ had central obesity, $77.9 \%$ had hypertension, and $59.2 \%$ had dyslipidemia. In addition, 30.0\% abused alcohol and 7.4\% smoked cigarette. In general terms, glycemic control was poorer among male T2DM subjects showing poorer controls compared to females. Females had higher prevalence of some important vascular risk factors. This is explained by the fact that female T2DM patients especially those who are menopausal have worse CVD risk factor profiles and mortalities compared to their male counterparts $[28,29]$. This was illustrated by a meta-analysis of 37 
TABLE 1: Demographic and clinical characteristics of 780 T2DM participants enrolled for the study.

\begin{tabular}{|c|c|c|c|c|}
\hline Test parameters & Total & Male & Female & $p$ value \\
\hline Number (\%) & $780(100)$ & $330(42.3)$ & $450(57.7)$ & \\
\hline Age, mean \pm SD & $57.4 \pm 9.4$ & $57.8 \pm 9.5$ & $57.3 \pm 9.3$ & 0.65 \\
\hline Age at diagnosis, median (IQR) & $50(43-56)$ & $51(43-60)$ & $50(43-78)$ & 0.08 \\
\hline Duration of diabetes, mean \pm SD & $9.8 \pm 5.6$ & $9.9 \pm 3.2$ & $9.6 \pm 8.9$ & 0.09 \\
\hline Body mass index (kg/m), median (IQR) & $27.5(24.8-30.9)$ & $27.0(24-30.3)$ & $27.9(25.1-31.5)$ & $0.04^{*}$ \\
\hline General obesity $(\%)$ & $262(33.6)$ & $92(27.9)$ & $170(37.8)$ & $0.04^{*}$ \\
\hline Waist circumference, $\mathrm{cm}$, median (IQR) & $97(87-105)$ & $94(84-101)$ & $98(90-106)$ & $<0.001^{*}$ \\
\hline Central obesity, $n(\%)$ & $582(74.6)$ & $156(47.3)$ & $426(94.7)$ & $<0.001^{*}$ \\
\hline Fasting blood glucose, median (IQR) & $8.8(6.7-12.6)$ & $8.6(6.7-12.2)$ & $9.1(6.8-13.5)$ & 0.4 \\
\hline Hbalc (\%), median (IQR) & $8.1(6.8-9.6)$ & $8.1(6.6-9.8)$ & $8.1(6.9-9.6)$ & 0.92 \\
\hline Abnormal glycemic control (>7\%) & $716(66.2)$ & $220(66.7)$ & $286(63.6)$ & 0.85 \\
\hline Total cholesterol mmol/L, median (IQR) & $5.0(4.1-6.0)$ & $5(4.2-5.9)$ & $5(4-6.1)$ & 0.63 \\
\hline Hypercholesterolemia, $n$ (\%) & $400(51.3)$ & $164(49.7)$ & $236(52.4)$ & 0.15 \\
\hline Triglycerides (mmol/L), median (IQR) & $1.2(0.9-1.6)$ & $1.16(0.85-1.6)$ & $1.24(0.94-1.57)$ & 0.22 \\
\hline Hypertriglyceridemia, $n$ (\%) & $170(21.8)$ & $66(20.0)$ & $104(23.1)$ & 0.44 \\
\hline LDL-C, median (IQR) & $3.2(2.1-3.9)$ & $3.1(2.2-3.8)$ & $3.2(2.1-4.0)$ & 0.92 \\
\hline High LDL, $n(\%)$ & $470(60.3)$ & $186(56.4)$ & $284(63.1)$ & 0.18 \\
\hline HDL-C, median (IQR) & $1.2(1-1.5)$ & $1.1(1-1.4)$ & $1.2(1.0-1.5)$ & 0.2 \\
\hline Low HDL-C, $n(\%)$ & $54(6.9)$ & $32(9.6)$ & $22(4.9)$ & $0.007^{*}$ \\
\hline Dyslipidemia, $n(\%)$ & $462(59.2)$ & $196(59.4)$ & $266(59.1)$ & 0.12 \\
\hline Systolic blood pressure, median (IQR) & $140(130-150)$ & $140(129-150)$ & $140(130-150)$ & 0.43 \\
\hline Diastolic blood pressure, median (IQR) & $80(70-90)$ & $80(70-90)$ & $80(80-90)$ & $0.01^{*}$ \\
\hline Hypertension, $n(\%)$ & $602(77.9)$ & $272(82.4)$ & $330(73.3)$ & $0.03^{*}$ \\
\hline Alcohol, $n(\%)$ & $234(30.0)$ & $200(60.6)$ & $34(7.6)$ & $<0.001^{*}$ \\
\hline Current smokers, $n(\%)$ & $58(7.4)$ & $56(17.0)$ & $2(0.4)$ & $<0.001^{*}$ \\
\hline Framingham CHD risk, \%, median (IQR) & $6(2-12)$ & $12(8-20)$ & $3(1-6)$ & $<0.001^{*}$ \\
\hline UKPDS CHD risk score, median (IQR) & $4(0.4-7)$ & $9.4(5.7-13.4)$ & $5.8(3.4-9.6)$ & $<0.001^{*}$ \\
\hline Subclinical hypothyroidism & $64(8.2)$ & $14(4.2)$ & $50(11.1)$ & $0.02^{*}$ \\
\hline Subclinical hyperthyroidism & $22(2.8)$ & $14(4.2)$ & $8(1.8)$ & $<0.001$ \\
\hline Hypothyroidism & $12(1.6)$ & $2(0.6)$ & $10(2.2)$ & 0.08 \\
\hline Hyperthyroidism & $4(0.5)$ & $2(0.6)$ & $2(0.4)$ & 0.21 \\
\hline Euthyroidism & $678(86.9)$ & $298(90.3)$ & $380(84.4)$ & 0.02 \\
\hline
\end{tabular}

CHD: coronary heart disease; HbAlc: glycated hemoglobin; HDL-C: high-density lipoprotein cholesterol; IQR: interquartile range; LDL-C: low-density lipoprotein cholesterol; UKPDS: United Kingdom Prospective Diabetes Study; SD: standard deviation. ${ }^{*} p<0.05$ statistically significant.

prospective cohort studies where the risk of fatal CHD was $50 \%$ higher in female T2DM patients compared to males [30]. The reason for this postmenopausal T2DM female predilection towards increased CHD risk is multifactorial. Principally, it has been demonstrated that chronic hyperglycemia alters the estrogen-related protective mechanisms that leave premenopausal woman at a lower CVD risk compared to males. This leads to notable adverse changes in CVD risk factors leading to enhanced atherogenesis in females even premenopausal [31,32]. Additionally, postmenopausal T2DM females have been shown to possess a relatively heavier risk factor burden [32] as well as a significant array and involvement of inflammatory factors [33], smaller size of coronary vessels, and disordered angiogenesis [34, 35]. The removal of the protective effect of female sex on the risk of $\mathrm{CHD}$ in people with diabetes warrants aggressive screening and treatment of females with this risk [36].

The proportion of patients with high CHD risk (18.7\%) recorded in this present study is comparable to that found in similar studies involving African American [37] and non-Hispanic white T2DM subjects [38] who were assessed using the Framingham risk scoring. However, among Brazilian T2DM subjects where UKPDS was used in risk assessment, a significantly higher CHD risk was recorded [39]. It is unknown whether these differences observed in $\mathrm{CHD}$ risk prevalence are due to the different methods used 
TABLE 2: Demographic and clinical characteristics of T2DM participants based on CHD risk.

\begin{tabular}{|c|c|c|c|c|c|}
\hline Test parameters & Total & Low & $\begin{array}{l}\text { CHD risk } \\
\text { Moderate }\end{array}$ & High & $p$ \\
\hline Number (\%) & $780(100)$ & $424(54.4)$ & $210(26.9)$ & $146(18.7)$ & \\
\hline Age, mean $\pm S D$ & $59.2 \pm 8.6$ & $56.6 \pm 7.9$ & $61.6 \pm 8.5$ & $62.9 \pm 8.4$ & $<0.0001^{*}$ \\
\hline Female gender, $n(\%)$ & $450(57.7)$ & $326(76.9)$ & $124(59.0)$ & $52(35.6)$ & $<0.0001^{*}$ \\
\hline Age at diagnosis, mean \pm SD & $51.3 \pm 9.0$ & $47.4 \pm 7.2$ & $52.8 \pm 7.9$ & $54.2 \pm 10.2$ & $<0.0004^{*}$ \\
\hline BMI, median (IQR) & $27(24.1-30.8)$ & $27(24-29.5)$ & $26.7(23-30.9)$ & $27(24-30.9)$ & 0.37 \\
\hline Waist circumference, median (IQR) & $98(88-108)$ & $87.3(95.5-105)$ & $107(99-114)$ & $95(85-109)$ & $<0.0001^{*}$ \\
\hline Central obesity, $n(\%)$ & $582(74.6)$ & $256(60.4)$ & $190(90.5)$ & $136(93.2)$ & $<0.0001^{*}$ \\
\hline Fasting glucose, median (IQR) & $8.6(6.7-13.0)$ & $8.2(6.7-12.9)$ & $8.6(6-12.5)$ & $9.4(7.1-14)$ & 0.37 \\
\hline Hbalc (\%), median (IQR) & $8.2(7-9.8)$ & $7.9(6.9-9.5)$ & $8.7(6.9-9.9)$ & $8.4(7.3-10)$ & $<0.0001^{*}$ \\
\hline Abnormal glycemic control (>7\%) & $516(66.2)$ & $232(54.7)$ & $160(76.2)$ & $124(84.9)$ & $<0.001^{*}$ \\
\hline Total cholesterol, median (IQR) & $5.3(4.3-6.65)$ & $5.0(4.1-5.9)$ & $5.7(4.7-6.9)$ & $6.1(6-7.9)$ & $<0.0001^{*}$ \\
\hline Hypercholesterolemia, $n(\%)$ & $400(51.3)$ & $196(46.2)$ & $112(53.3)$ & $92(63.0)$ & $<0.0001^{*}$ \\
\hline Triglycerides, median (IQR) & $0.95(1.22-1.6)$ & $1.2(0.9-1.5)$ & $1.2(1.0-1.7)$ & $1.4(1.0-1.7)$ & 0.06 \\
\hline LDL-C, median (IQR) & $3.3(2.3-4.2)$ & $3.2(2.2-3.8)$ & $3.6(2.6-4.4)$ & $4.19(3.2-5)$ & $<0.0001^{*}$ \\
\hline High LDL, $n(\%)$ & $470(60.3)$ & $216(50.9)$ & $136(64.8)$ & $118(80.8)$ & $<0.0001^{*}$ \\
\hline HDL-C, median (IQR) & $1.2(1-1.43)$ & $1.2(1-1.5)$ & $1.2(1-1.4)$ & $1.1(1-1.32)$ & 0.14 \\
\hline Low HDL-C, $n(\%)$ & $108(13.8)$ & $26(6.1)$ & $38(18.1)$ & $44(30.1)$ & $<0.0001^{*}$ \\
\hline Dyslipidemia, $n(\%)$ & $462(59.2)$ & $212(50.0)$ & $124(59.1)$ & $126(86.3)$ & $<0.00001^{*}$ \\
\hline Systolic blood pressure, median (IQR) & $140(130-152)$ & $140(130-150)$ & $140(130-154)$ & $148(130-160)$ & 0.14 \\
\hline Diastolic blood pressure, median (IQR) & $80(80-90)$ & $80(80-90)$ & $80(70-90)$ & $80(80-90)$ & 0.78 \\
\hline Hypertension, $n(\%)$ & $602(77.9)$ & $304(71.7)$ & $158(75.2)$ & $140(95.9)$ & $<0.001^{*}$ \\
\hline Alcohol, $n(\%)$ & $234(30.0)$ & $108(25.5)$ & $62(29.5)$ & $64(43.8)$ & $<0.0001^{*}$ \\
\hline Current smokers, $n(\%)$ & $58(7.4)$ & $26(6.1)$ & $16(7.6)$ & $18(12.3)$ & $<0.0001^{*}$ \\
\hline
\end{tabular}

CHD: coronary heart disease; HbAlc: glycated hemoglobin; HDL-C: high-density lipoprotein cholesterol; IQR: interquartile range, LDL-C: low-density lipoprotein cholesterol; SD: standard deviation. ${ }^{*} p<0.05$ statistically significant.

for assessment and study sample sizes. A high risk of CHD was seen among Caucasians and South Americans $[38,39]$. This applies particularly to men, where both morbidity and mortality from heart disease are about half that of the general population [40]. Data on African Americans from the United States conflict this finding, showing higher $\mathrm{CHD}$ rates than US whites [41]. This appears to be in part explained by the degree of acculturation and miscegenation, as birthplace within or outside the USA was a strong determinant of mortality risk [35]. Furthermore, African men have been shown to be less centrally obese than their Caucasian counterparts [37].

Thus, with this present study, which to the best of our knowledge is the first among West Africans, we have shown a higher CHD risk in T2DM subjects with thyroid dysfunction compared with those who were euthyroid. This we attribute to the significantly higher risk factor burden of CHD seen in T2DM subjects with thyroid dysfunction compared to their euthyroid counterparts recorded in the study. Moreover, this effect is better explained by the higher CHD risk seen among patients with hypothyroidism compared to those with other forms of thyroid dysfunction and euthyroidism. It is well known that patients with hypothyroidism possess significant incidence of inflammatory factors, hypercholesterolemia, and diastolic blood pressures compared to those with other forms of thyroid dysfunction [38].

A significantly positive linear association found in T2DM subjects between TSH and CHD risk could be attributed to the increasing association of thyroid dysfunction with high inflammatory markers, dyslipidemia, and poor glycemic control [39]. It has been postulated that high inflammatory state induced by thyroid dysfunction produces oxidative stress, insulin resistance, cell apoptosis, vascular endothelial injury, platelet activation, and neuroendocrine disorders which all promote the development of atherosclerosis $[40,41]$. For this reason, it is now recognised that subclinical hypothyroidism together with hypothyroidism are risk factors for increased CHD risk and mortality in the general population as well as diabetics even after accounting for traditional vascular risk factors [42].

Some inherent limitations were associated with this study including our sample size composition. We had fewer males with T2DM relative to females which may have influenced 


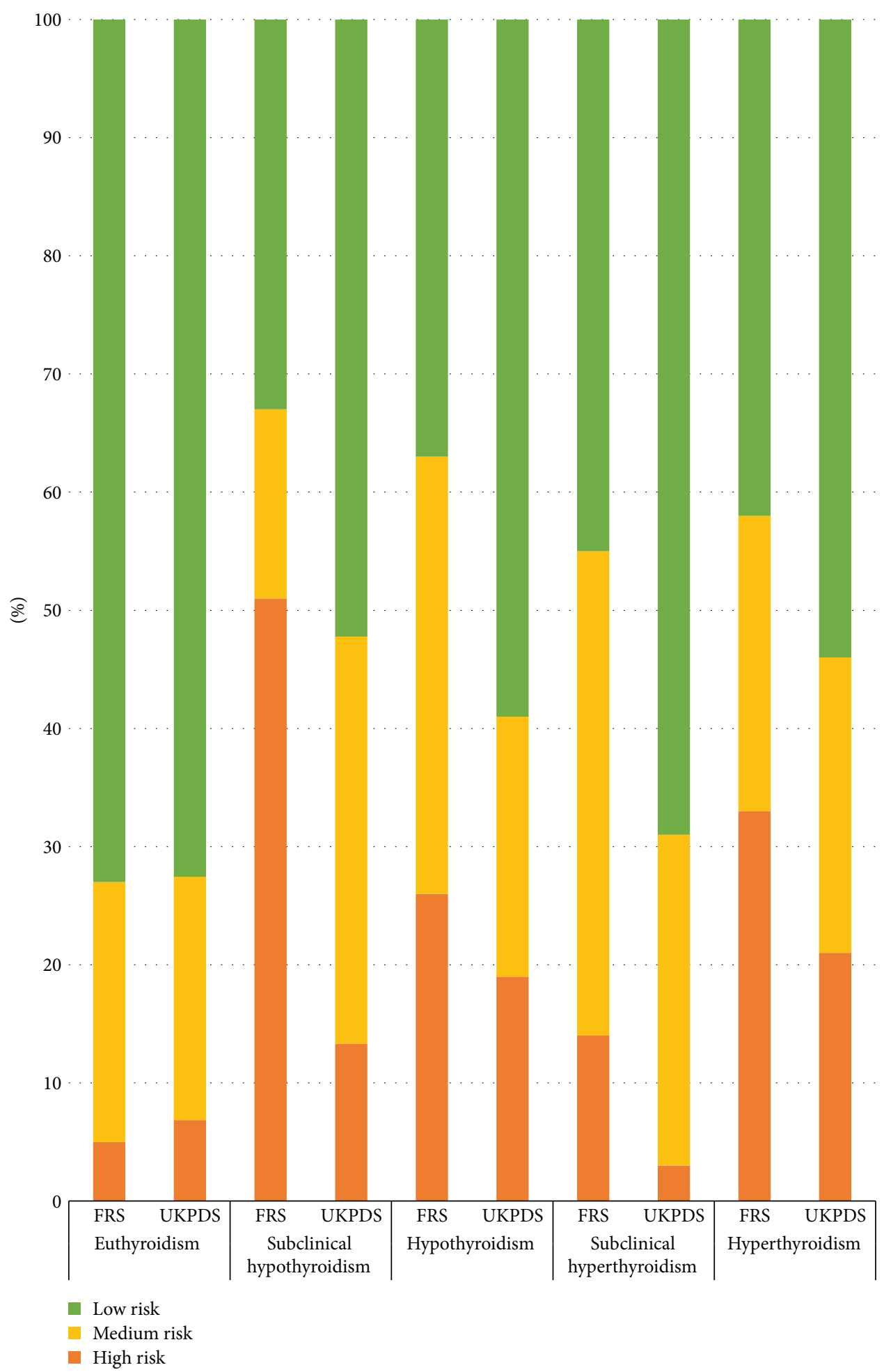

FIgURE 1: Variation of coronary heart disease risk per Framingham and UKPDS Scores.

the results. Moreover, confounders such as physical activity and dietary patterns were not explored in this study even though they could have influenced the CHD risk. Secondly, the cross-sectional study design means that the associations observed between thyroid dysfunction and CHD risk could not be interpreted as causal relationships.

\section{Conclusion}

We conclude that our T2DM patients with thyroid dysfunction compared to their euthyroid counterparts have a significantly higher CHD risk. A significant association of CHD risk has been shown with $\mathrm{HbAlc}$ and dyslipidemia. 
TABle 3: Pearson bivariate correlation between ten-year coronary heart disease risk and independent variables.

\begin{tabular}{lcc}
\hline Test parameters & Framingham & UKPDS \\
\hline Waist circumference & $0.12(0.061)$ & $-0.08(0.42)$ \\
Glycated hemoglobin, HbA1c & $0.51(0.041)$ & $0.42(<0.0001)$ \\
Total cholesterol & $0.49(0.0001)$ & $0.14(<0.51)$ \\
Low-density lipoprotein & $0.37(<0.0001)$ & $0.10(0.04)$ \\
cholesterol & & \\
$\begin{array}{l}\text { High-density lipoprotein } \\
\text { cholesterol }\end{array}$ & $0.02(0.21)$ & $-0.06(0.12)$ \\
Triglycerides & $0.12(0.02)$ & $0.11(0.06)$ \\
Systolic blood pressure & $0.12(0.12)$ & $0.10(0.04)$ \\
Thyroid stimulating hormone & $0.27(0.0006)$ & $0.32(0.02)$ \\
Free triiodothyronine & $0.07(0.23)$ & $0.10(0.34)$ \\
Free thyroxine & $0.11(0.09)$ & $0.13(0.15)$ \\
\hline
\end{tabular}

Physicians taking care of these patients should have a high index of suspicion for thyroid dysfunction in patients with high CVD risk.

\section{Abbreviations}

CHD: $\quad$ Coronary heart disease

CVD: $\quad$ Cardiovascular disease

FT3: $\quad$ Free triiodothyronine

FT4: $\quad$ Free thyroxine

HbAlc: Glycated hemoglobin

HDL: High-density lipoprotein cholesterol

LDL-C: Low-density lipoprotein cholesterol

TC: $\quad$ Total cholesterol

TG: $\quad$ Triglycerides

T2DM: Type 2 diabetes mellitus

UKPDS: United Kingdom Prospective Diabetes Study.

\section{Ethical Approval}

The study was approved by the Committee for Human Research Publications and Ethics at the School of Medical Sciences, Kwame Nkrumah University of Science and Technology, and the Komfo Anokye Teaching Hospital, Kumasi.

\section{Consent}

All participants gave an informed consent with those unable to understand or sign the informed consent excluded.

\section{Conflicts of Interest}

There is no competing interest involving any of the authors of this study.

\section{Authors' Contributions}

Osei Sarfo-Kantanka conceived the study, participated in its design, and drafted the manuscript. Fred Stephen Sarfo contributed in the study design and helped in the manuscript preparation and statistical analysis. Eunice Oparebea
Ansah contributed in conducting the field and manuscript preparation. Ishmael Kyei participated in the manuscript preparation and helped in the analysis of data.

\section{Acknowledgments}

The authors acknowledge the immense role played by the nurses and laboratory scientists at the Diabetes Clinic, KATH. The authors also appreciate the contribution of the staff at the office of the Department of Medicine, KATH, who helped in typing the manuscript. The authors are eternally grateful to all participants for participating in the study. This study was funded by Osei Sarfo-Kantanka.

\section{References}

[1] "International Diabetes Federation IDF diabetes atlas," March 2017, http://www.diabetesatlas.org/resources/2015-atlas.htlm.

[2] L. Chen, D. J. Magliano, and P. Z. Zimmet, "The worldwide epidemiology of type 2 diabetes mellitus-present and future perspectives," Nature Reviews Endocrinology, vol. 8, no. 4, pp. 228-236, 2012.

[3] WHO, "Global status report on non-communicable diseases 2010," March 2017, http://www.who.int/nmh/publications/ ncd_report2010/en/.

[4] A. G. Amoah, S. K. Owusu, and S. Adjei, "Diabetes in Ghana: a community-based prevalence study in Greater Accra," Diabetes Research and Clinical Practice, vol. 56, no. 3, pp. 197-205, 2002.

[5] S. M. Haffner, S. Lehto, T. Ronnemaa, K. Pyörälä, and M. Laakso, "Mortality from coronary heart disease in subjects with type 2 diabetes and non-diabetic subjects with and without prior myocardial infarction," The New England Journal of Medicine, vol. 339, no. 4, pp. 229-234, 1998.

[6] A. Juutilainen, S. Lehto, T. Rönnemaa, K. Pyörälä, and M. Laakso, "Type 2 diabetes as a "coronary heart disease equivalent": an 18-year prospective population-based study in Finnish subjects," Diabetes Care, vol. 28, no. 12, pp. 29012907, 2005.

[7] J. Stamler, O. Vaccaro, J. D. Neaton, D. Wentworth, and The Multiple Risk Factor Intervention Trial Research Group, "Diabetes, other risk factors, and 12-yr cardiovascular mortality for men screened in the multiple risk factor intervention trial," Diabetes Care, vol. 16, no. 2, pp. 434-444, 1993.

[8] Diabetes drafting group, "Prevalence of small vessel disease and large vessel disease in diabetic patients from 14 centers. The World Health Organization Multinational Study of Vascular Disease in diabetics," Diabetologia, vol. 28, Supplement 1, pp. 615-640, 1985.

[9] R. Sathish and V. Mohan, "Diabetes and thyroid diseasesa review," International Journal of Diabetes in Developing Countries, vol. 23, no. 4, pp. 120-123, 2003.

[10] S. Danzi and I. Klein, "Thyroid hormone and blood pressure regulation," Current Hypertension Reports, vol. 5, no. 6, pp. 513-520, 2003.

[11] E. A. Palmieri, S. Fazio, G. Lombardi, and B. Biondi, "Subclinical hypothyroidism and cardiovascular risk: a reason to treat?," Treatments in Endocrinology, vol. 3, no. 4, pp. 233244, 2004.

[12] S. A. Chubb, W. A. Davis, and Z. Inman, "Prevalence and progression of subclinical hypothyroidism in women with 
type 2 diabetes: the Fremantle diabetes study," Clinical Endocrinology, vol. 62, no. 4, pp. 480-486, 2005.

[13] P. Haentjens, A. Van Meerhaeghe, K. Poppe, and B. Velkeniers, "Subclinical thyroid dysfunction and mortality: an estimate of relative and absolute excess all-cause mortality based on time-to-event data from cohort studies," European Journal of Endocrinology, vol. 159, no. 3, pp. 329-341, 2008.

[14] T. Sathyapalan, A. M. Manuchehri, A. S. Rigby, and S. L. Atkin, "Subclinical hypothyroidism is associated with reduced allcause mortality in patients with type 2 diabetes," Diabetes Care, vol. 33, no. 3, article e37, 2010.

[15] N. Ochs, R. Auer, D. C. Bauer et al., "Meta-analysis: subclinical thyroid dysfunction and the risk of coronary heart disease and mortality," Annals of Internal Medicine, vol. 148, no. 11, pp. 832-845, 2008.

[16] S. Razvi, A. Shakoor, M. Vanderpump, J. U. Weaver, and S. H. S. Pearce, "The influence of age on the relationship between subclinical hypothyroidism and ischemic heart disease: a metaanalysis," The Journal of Clinical Endocrinology \& Metabolism, vol. 93, no. 8, pp. 2998-3007, 2008.

[17] F. J. del Canizo-Gomez and M. N. Moreira-Andres, "Stick control of modifiable risk factors for type 2 diabetes," The New England Journal of Medicine, vol. 348, no. 5, pp. 383-393, 2003.

[18] R. B. D’Agostino Sr., R. S. Vasan, M. J. Pencina et al., “General cardiovascular risk profile for use in primary care: the Framingham heart study," Circulation, vol. 117, no. 6, pp. 743-753, 2008.

[19] The University of Oxford, Diabetes trial unit, "The Oxford Centre for Diabetes, Endocrinology and Metabolism, UKPDS risk engine," February 2017, http://www.dtu.ox.ac. uk/riskengine/download.php.

[20] WHO/NCD/NCS/99.2, WHO Consultation. Definition, Diagnosis and Classification of Diabetes Mellitus and Its Complications: Report of a WHO Consultation Part 1: Diagnosis and Classification of Diabetes Mellitus, World Health Organization, Geneva, 1999, Publication no. WHO/NCD/NCS/99.2.

[21] S. G. Wannamethee, A. G. Shaper, and I. J. Perry, "British regional heart study: smoking as a modifiable risk factor for type 2 diabetes in middle-aged men," Diabetes Care, vol. 204, no. 9, pp. 1590-1595, 2001.

[22] A. G. Shaper, G. Wanathee, and M. Walker, "Alcohol and mortality in British: explaining the $\mathrm{U}$ shaped curve," The Lancet, vol. 332, no. 8623, pp. 1267-1273, 1988.

[23] World Health Organisation, Obesity: Prevention and Managing the Global Epidemic. Report of a WHO Consultation, WHO Technical Report Series 894, Geneva, 2000, http://www.who .int/nutrition/publications/obesity/WHO_TRS_894/en/.

[24] J. Chalmers, S. McMahon, G. Mancia et al., "1999 World Health Organisation-International Society of Hypertension Guidelines for treatment of hypertension," Clinical and Experimental Hypertension, vol. 21, no. 5-6, pp. 1009-1060, 1999.

[25] O. Sarfo-Kantanka, F. S. Sarfo, E. O. Ansah et al., "Frequency and determinants of thyroid autoimmunity in Ghanaian type 2 diabetes patients: a case-control study," BMC Endocrine Disorders, vol. 17, no. 1, p. 2, 2017.

[26] P. W. Wilson, R. B. D’Agostino, D. Levy, A. M. Belanger, H. Silbershatz, and W. B. Kannel, "Prediction of coronary heart disease using risk factor categories," Circulation, vol. 97, no. 18, pp. 1837-1847, 1998.

[27] S. N. Darko, D. D. Yar, E. Owusu-Dabo et al., "Variations in levels of IL- 6 and TNF- $\alpha$ in type 2 diabetes mellitus between rural and urban Ashanti Region of Ghana," BMC Endocrine Disorders, vol. 15, no. 1, p. 50, 2015.

[28] G. Hu, P. Jousilahti, Q. Qiao, S. Katoh, and J. Tuomilehto, "Sex differences in cardiovascular and total mortality among diabetic and non-diabetic individuals with or without a history of myocardial infarction," Diabetologia, vol. 48 , no. 5, pp. 856-861, 2005.

[29] W. L. Lee, A. M. Cheung, D. Cape, and B. Zinman, "Impact of diabetes on coronary artery disease in women and men: a meta-analysis of prospective studies," Diabetes Care, vol. 23, no. 7, pp. 962-968, 2000.

[30] R. Huxley, F. Barzi, and M. Woodward, "Excess risk of coronary heart diseases associated with diabetes in men and women: meta-analysis of 37 prospective cohort studies," BMJ, vol. 332, no. 7533, pp. 73-78, 2006.

[31] I. Martin-Timon, C. Sevillano- Collantes, A. Sejura-Galindo, and F. J. Del Cañizo-Gómez, "Type 2 diabetes and cardiovascular disease: have all the risk factors the same strength?," World Journal of Diabetes, vol. 5, no. 4, pp. 444-470, 2014.

[32] H. O. Steinberg, G. Paradisi, J. Cronin et al., “Type II diabetes abrogates sex differences in endothelial function in premenopausal women," Circulation, vol. 101, no. 17, pp. 2040 2046, 2000.

[33] A. M. Kanaya, D. Grady, and E. Barrett-Connor, "Explaining the sex difference in coronary heart disease mortality among patients with type 2 diabetes mellitus: a meta-analysis," Archives of Internal Medicine, vol. 162, no. 15, pp. 17371745, 2002.

[34] A. A. Rivellese, G. Riccardi, and O. Vaccaro, "Cardiovascular risk in women with diabetes," Nutrition, Metabolism \& Cardiovascular Diseases, vol. 20, no. 6, pp. 474-480, 2010.

[35] C. S. Fox, "Cardiovascular disease risk factors, type 2 diabetes mellitus and the Framingham free heart study," Trends in Cardiovascular Medicine, vol. 20, no. 3, pp. 90-95, 2010.

[36] A. R. Cappola, L. P. Fried, A. M. Arnold et al., "Thyroid status, cardiovascular risk, and mortality in older adults," JAMA, vol. 295, no. 9, pp. 1033-1041, 2006.

[37] A. Adeniye, A. R. Folson, F. Z. Brancati, M. Desvorieux, J. S. Pankow, and H. Taylor, "Incidence and risk factors of African American with diabetes: atherosclerosis risk in communities (ARIC) study," Journal of the National Medical Association, vol. 94, no. 12, pp. 1025-1035, 2002.

[38] G. Iervasi, S. Molinaro, P. Landi et al., "Association between increased mortality and mild thyroid dysfunction in cardiac patients," Archives of Internal Medicine, vol. 167, no. 14, pp. 1526-1532, 2007.

[39] M. B. Gomes, D. Giannella-Neto, M. Faria et al., "Estimating cardiovascular risk in patients with type 2 diabetes: a national multicenter study in Brazil," Diabetology \& Metabolic Syndrome, vol. 1, no. 1, p. 22, 2009.

[40] M. Laakso, "Cardiovascular disease in type 2 diabetes from population to man to mechanisms," Diabetes Care, vol. 33, no. 2, pp. 442-449, 2010.

[41] S. Y. Gebreab and A. V. Diez Roux, "Exploring racial disparities in CHD mortality between blacks and whites across the United States: a geographically weighted regression approach," Health \& Place, vol. 18, no. 5, pp. 1006-1014, 2012.

[42] I. L. G. Todorova, K. L. Tucker, M. P. Jimenez, A. K. Lincoln, S. Arevalo, and L. M. Falcón, "Determinants of self-rated health and the role of acculturation: implications for health inequalities," Ethnicity \& Health, vol. 18, no. 6, pp. 563-585, 2013. 


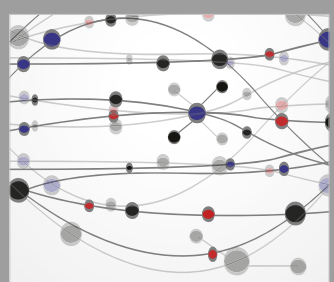

The Scientific World Journal
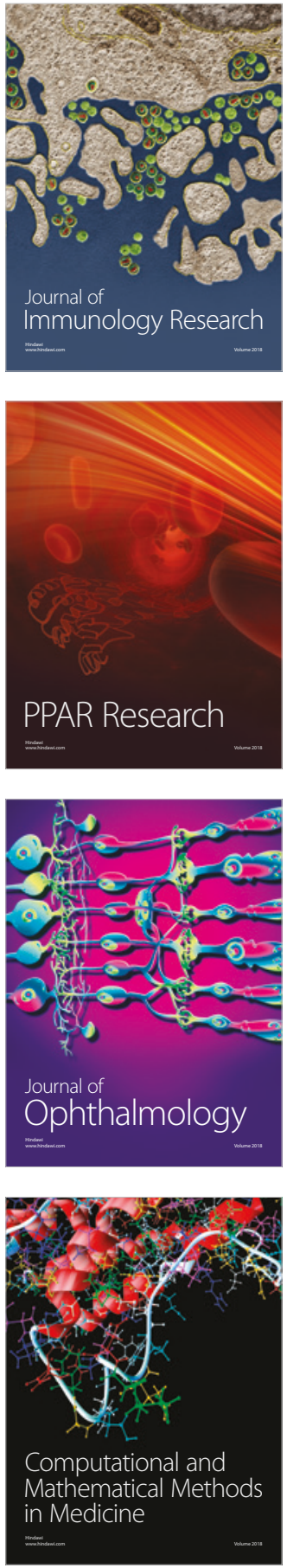

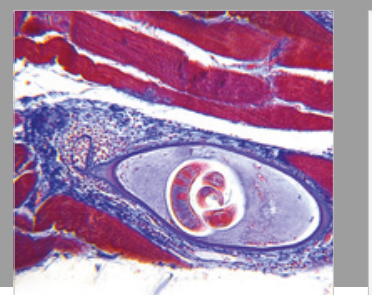

Gastroenterology Research and Practice

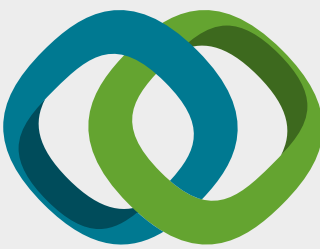

\section{Hindawi}

Submit your manuscripts at

www.hindawi.com
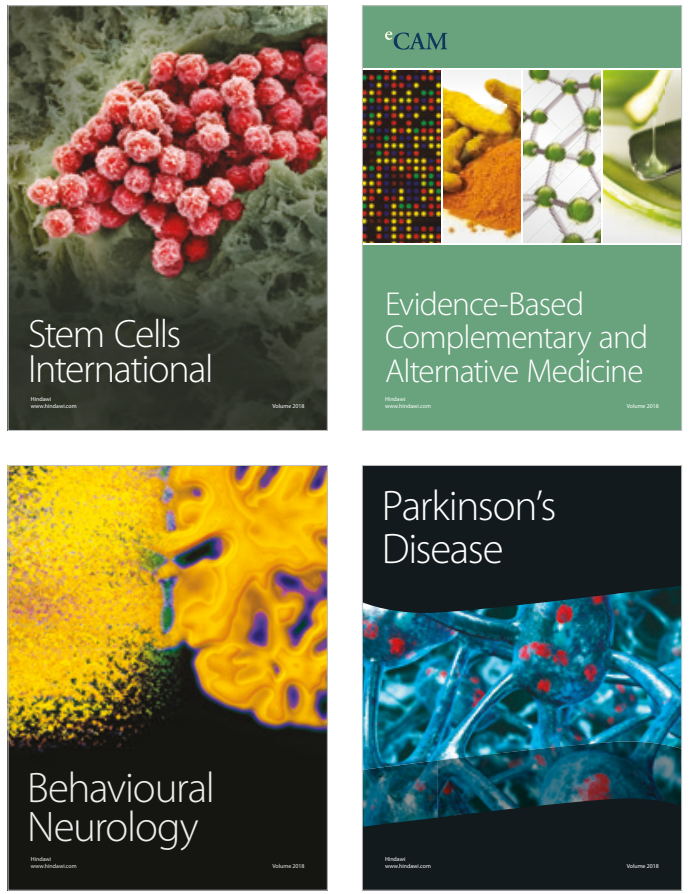

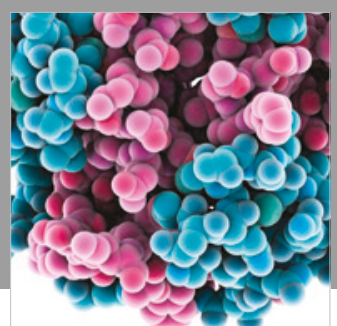

ournal of

Diabetes Research

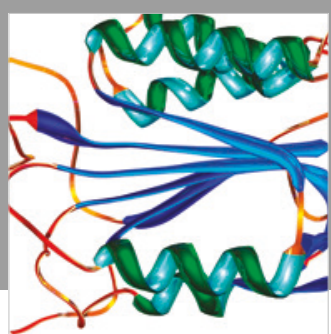

Disease Markers
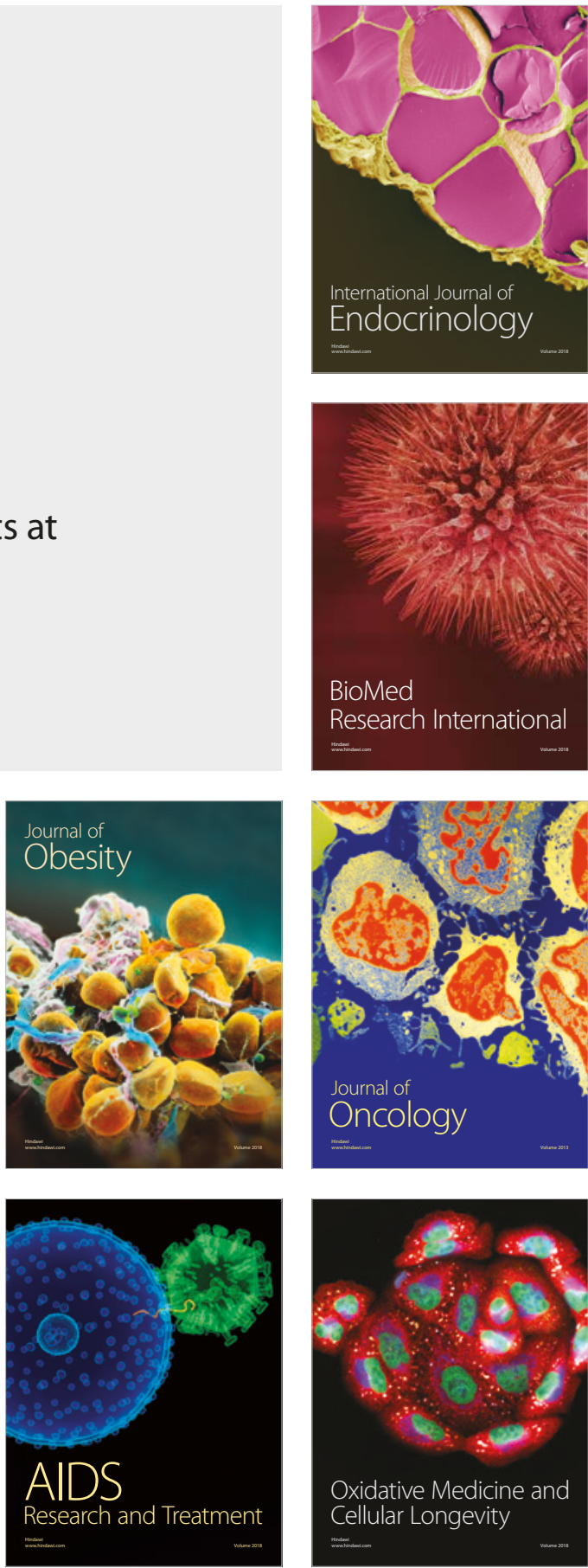